\title{
Video Article \\ A Rabbit Model of Aqueous-Deficient Dry Eye Disease Induced by Concanavalin A Injection into the Lacrimal Glands: Application to Drug Efficacy Studies
}

\author{
Robert A. Honkanen ${ }^{1}$, Liqun Huang ${ }^{2,3}$, Basil Rigas ${ }^{2}$ \\ ${ }^{1}$ Department of Ophthalmology, Stony Brook University \\ ${ }^{2}$ Department of Preventive Medicine, Stony Brook University \\ ${ }^{3}$ Medicon Pharmaceuticals, Inc.
}

Correspondence to: Robert A. Honkanen at Robert.Honkanen@stonybrookmedicine.edu

URL: https://www.jove.com/video/59631

DOI: doi:10.3791/59631

Keywords: Medicine, Issue 155, dry eye, dry eye disease, dry eye syndrome, rabbit model, Concanavalin A, tear break-up time, Schirmer's tear test, tear osmolarity, rose bengal staining, ocular drug development

Date Published: $1 / 24 / 2020$

Citation: Honkanen, R.A., Huang, L., Rigas, B. A Rabbit Model of Aqueous-Deficient Dry Eye Disease Induced by Concanavalin A Injection into the Lacrimal Glands: Application to Drug Efficacy Studies. J. Vis. Exp. (155), e59631, doi:10.3791/59631 (2020).

\section{Abstract}

Dry eye disease (DED), a multifactorial inflammatory disease of the ocular surface, affects 1 in 6 humans worldwide with staggering implications for quality of life and health care costs. The lack of informative animal models that recapitulate its key features impedes the search for new therapeutic agents for DED. Available DED animal models have limited reproducibility and efficacy. A model is presented here in which DED is induced by injecting the mitogen concanavalin $A(\operatorname{Con} A)$ into the orbital lacrimal glands of rabbits. Innovative aspects of this model are the use of ultrasound (US) guidance to ensure optimal and reproducible injection of Con A into the inferior lacrimal gland; injection of Con A into all orbital lacrimal glands that limits compensatory production of tears; and use of periodic repeat injections of Con A that prolong the state of DED at will. DED and its response to test agents are monitored with a panel of parameters that assess tear production, the stability of the tear film, and the status of the corneal and conjunctival mucosa. They include tear osmolarity, tear break-up time, Schirmer's tear test, rose bengal staining, and tear lactoferrin levels. The induction of DED and the monitoring of its parameters are described in detail. This model is simple, robust, reproducible, and informative. This animal model is suitable for the study of tear physiology and of the pathophysiology of DED as well as for the assessment of the efficacy and safety of candidate agents for the treatment of DED.

\section{Video Link}

The video component of this article can be found at https://www.jove.com/video/59631/

\section{Introduction}

Dry eye disease (DED) is a chronic condition with high prevalence and morbidity ${ }^{1,2,3,4}$. Inflammation plays a key role in its pathogenesis ${ }^{5,6}$. The pathophysiology of DED is conceptualized as deriving from either under-production or over-evaporation of tears; the former is also known as aqueous-deficient $D_{E D}{ }^{7}$. Sjögren's syndrome, an extensively studied prototypical cause of DED, affects primarily the lacrimal glands (LGs) and is a striking example of their importance in the pathogenesis of DED. DED is often treated with artificial tears which provide temporary relief, or with cyclosporine or lifitegrast, both of which suppress ocular inflammation. None of the available treatments for DED are optimal, necessitating the development of new agents ${ }^{8,9}$.

The search for new therapeutic agents for DED is hampered by three major challenges: the lack of a recognized druggable molecular target, which may be elusive given the pathophysiological complexity of DED; the sparsity of promising agents; and the lack of animal models that recapitulate key features of DED.

As with most drug development efforts, informative animal models of DED are a crucial investigative tool, notwithstanding the axiomatic statement that no animal model completely recapitulates a human disease. Mouse, rat, and rabbit models of DED are the most commonly used while dogs and primates are used infrequently ${ }^{10,11}$. Most of the more than 12 rabbit DED models reported to date attempt to reduce tear production by either removing LGs or by impeding their function ${ }^{12,13,14,15,16}$. Such approaches include the surgical resection of the ILG; closure of its excretory duct; and impairing LG function by irradiation or injection of one of the following: activated lymphocytes, mitogens, botulinum toxin, atropine, or benzalklonium. Major limitations of these methods are their inconsistency and the frequent partial suppression of tear production.

Concanavalin A (Con A), a lectin of plant origin, is a potent stimulator T-cell subsets and has been used in experimental models of hepatitis ${ }^{17}$ and $D E D^{18}$. The original Con A-based model was reported to offer significant advantages, including its relative simplicity; inflammatory cell influx in the LGs, mimicking diseases such as Sjogren's; stimulation of the proinflammatory cytokines IL-1 $\beta$, IL-8, and TGF- $\beta 1$; reduced tear function 
monitored by measuring tear fluorescein clearance and tear break-up time (TBUT); and drug responsiveness shown for an anti-inflammatory corticosteroid.

When this promising method was applied, in addition to its advantages, limitations were identified that necessitated its overall revision and drastic improvements. Three critical shortcomings of the method are documented. First, the model was an acute one; the induced DED subsided after about 1 week. Second, the response of the animals was inconsistent. As demonstrated, in "blind" transcutaneous injections to the Inferior LG (ILG), Con A was delivered only randomly to the targeted gland. Detailed study of the anatomy of the ILG revealed that its size could vary as much as 4 -fold ${ }^{19}$ making such injections "hit-or-miss" efforts. Finally, even when the ILG was injected, the superior LG (SLG) frequently compensated for the reduced tear flow, making the model problematic.

These key limitations were overcome by introducing three modifications to the method, generating a superior animal model of DED. First, the injection of Con A into the ILG was performed under ultrasound (US) guidance, ensuring that Con A entered the gland. The success of the injection was confirmed by obtaining a post-injection US image, as shown in Figure 1. Second, to remove the compensatory tear contribution of the SLG, both the palpebral and orbital portions of this gland were injected with Con A. Finally, this acute model of DED was converted to a chronic one by repeated injections of Con A every 7-10 days. DED of 2 months' duration is readily achieved in these rabbits. The success of this approach has been amply documented ${ }^{19}$.

As already mentioned, an important application of animal models of DED is to determine the efficacy and safety of candidate therapeutic agents. The utility of this model was demonstrated by the study of phosphosulindac (OXT-328), a novel anti-inflammatory small molecule ${ }^{20,21}$ administered as eye drops. Its efficacy was demonstrated based on a panel of parameters of DED ${ }^{19}$. The relative simplicity and informative nature of this model also allowed side-by-side comparison of phosphosulindac to the two FDA approved drugs for DED, cyclosporine and lifitegrast, demonstrating its strong preclinical superiority.

\section{Protocol}

All animal studies were approved by the Institutional Review Board of Stony Brook University and performed in accordance with the ARVO Statement for the Use of Animals in Ophthalmic and Vision Research.

\section{Animals and housing}

1. Acquire New Zealand White (NZW) rabbits weighing 2-3 kg.

2. House the rabbits singly in cages with strict temperature $\left(65 \pm 5^{\circ} \mathrm{F}\right)$ and humidity $(45 \pm 5 \%)$ control. Lighting should have a $12 \mathrm{~h}$ on/off cycle.

3. Provide unlimited access to water and standard rabbit chow. Eliminate dietary enrichments as they may contain vitamin $\mathrm{A}$ that affects the eye.

4. Acclimate the animals for at least 2 weeks prior to baseline measures or induction of dry eye.

\section{Methods of anesthesia and euthanasia}

NOTE: All procedures require mild sedation except for Con A injection that requires moderate sedation.

1. For mild sedation, inject acepromazine $(1 \mathrm{mg} / \mathrm{kg})$ subcutaneously over the shoulders using a 26-gauge needle. Endpoint for mild sedation: animals maintain a relaxed head position with ear lobes no longer fully upright.

NOTE: If the appropriate endpoint is not reached, an additional injection of acepromazine may be given. Animals should always remain awake, responsive to touching of their whiskers, and never show slowed breathing.

2. For moderate sedation, first give the animals acepromazine as above. After the endpoint is reached (see the note above), give isoflurane using a gas mask with $\mathrm{O}_{2}$ flow set at $1 \mathrm{~L} / \mathrm{min}$ and isoflurane delivery set to $5 \%$ (Figure 2).

3. Administer isoflurane until the rabbit's body tone is completely relaxed and ears are completely floppy. NOTE: No compensatory muscle movements should occur when the animal is turned on its side; breathing always remains spontaneous.

4. Spontaneous recovery occurs within 2-5 min: signs include spontaneous head movements and increased or normal muscular tone. After the experimental procedure is completed with moderate sedation, observe the rabbits for about 30 min or until their behavior returns to normal. NOTE: Ophthalmic ointment is not required during either form of sedation. 1) In mild sedation, animals are still alert and maintain a blink reflex. In moderate sedation, the inhibition of the blink reflex is so short that the ocular surface is not at risk. 2) Placement of ophthalmic ointment on the ocular surface precludes visualization of structures assessed during testing.

5. Euthanasia: Use an overdose of intravenous pentobarbital $(100 \mathrm{mg} / \mathrm{kg})$.

\section{Removal of nictitating membrane}

1. Perform the removal during the acclimation period (usually the first week) to allow complete and accurate evaluation of the cornea.

2. Injection to the right nictitating membrane

NOTE: If the nictitating membrane of both eyes is to be removed, it is simplest to do this in one session. Start with one eye and proceed as described. For clarity of description, this method starts with the right eye.

1. Place the rabbit in an appropriately sized restraining bag.

2. Induce mild sedation as described in step 2.1.

3. Apply $25 \mu \mathrm{L}$ of preservative-free lidocaine to the right eye using a micropipette.

4. Place a flexible wire lid speculum between the eyelids.

5. Using 0.3 forceps (or equivalent), grasp the nictitating membrane at its apex and extend it over the cornea.

6. Inject lidocaine $1 \%$ with 1:100,000 epinephrine subconjunctivally into the base of the nictitating membrane using a 26-gauge sharp needle (Figure 3A). A moderate bleb should form over the nictitating membrane. 
7. Remove the speculum.

3. Perform an identical injection to the left nictitating membrane.

4. Cutting the nictitating membrane

1. After approximately $5 \mathrm{~min}$, place the lid speculum back in the right eye. Grasp and retract the nictitating membrane at its apex using 0.3 forceps (or similar).

2. Cut the nictitating membrane off at its base using Westcott scissors or equivalent (Figure 3B).

NOTE: Bleeding is minimal and does not typically require cautery. Nevertheless, a high-temperature battery cautery is always kept nearby in case additional hemostasis is needed.

3. Remove the speculum.

4. Place topical antibiotic ointment on the eye (e.g., neomycin, polymyxin, bacitracin, and hydrocortisone).

5. Leave the Harderian gland intact. The Harderian gland is sometimes seen when the nictitating membrane is retracted. NOTE: If a large white mass or tissue elevation is seen in the nasal or superior subconjunctival region after the nictitating membrane is removed, the membrane was resected too close to its base allowing the Harderian gland to spontaneously prolapse. To prevent this in subsequent procedures, leave more of the nictitating membrane at the base.

6. Allow the ocular surface to heal for at least 1 week before further manipulations are made or ocular surface assays are performed.

\section{Measurement of dry eye parameters and collection of tear samples}

NOTE: Measure DED parameters based on study protocol needs (e.g., at baseline and specified time points thereafter). Measurements for DED should be done in the following order, with rigorous effort to faithfully replicate them each time. Test all animals at approximately the same time of day $( \pm 1 \mathrm{~h})$ to minimize circadian variations. These measurements usually require a team of two investigators.

1. Place the rabbit in a restraining bag. Induce mild sedation.

2. Tear Osmolarity ${ }^{22}$

1. Manually blink the eyelids 5-10 times to evenly distribute the tear layer on the ocular surface.

2. Gently retract the lower lid.

3. Sample tears with the TearLab Osmometer at the junction of the palpebral and bulbar conjunctiva along the lower fornix, just posterior to the base of the truncated nictitating membrane.

4. Measure osmolarity using the TearLab Osmolarity Test following the manufacturer's instructions.

3. Tear break-up time (TBUT)

1. Darken the room for this assay.

2. Place a wire lid speculum between the eyelids.

3. Apply a $50 \mu \mathrm{L}$ drop of $0.2 \%$ fluorescein over the corneal surface using a micropipette. If even distribution of the fluorescein over the cornea is not obtained with the first drop, place a second drop.

4. Immediately start a timer.

5. Observe the pre-corneal tear film under a blue light. The TBUT is defined as the time taken to develop black dots, lines or obvious disruption of the fluorescein film (Figure 4). If needed, use surgical loupes that provide +1.50 magnification to better visualize early signs of break-up. Monitor for up to $1 \mathrm{~min}$; if break-up as defined here occurs after $1 \mathrm{~min}$, record TBUT for only $60 \mathrm{~s}$.

4. Schirmer's Tear Test (STT)

1. Apply a $25 \mu \mathrm{L}$ drop of preservative-free lidocaine to the ocular surface.

2. Place a Weck cell surgical spear into the inferior fornix to absorb residual lidocaine and any tear fluid. If needed, use the lower eyelid to cover the proximal end of the sponge to help keep it in place (Figure 5A).

3. After approximately $30 \mathrm{~s}$, remove the Weck sponge.

4. Immediately insert a Schirmer's tear test strip into the space between the cornea and the palpebral conjunctiva at the mid-point of the lower lid.

5. Immediately start a timer (Figure 5B).

6. After 5 min, measure the length of the moistened portion of the strip; this is the STT value.

7. Perform measures in triplicate and report the average of the 3 readings as the STT value.

\section{Collection of tear samples}

1. To collect tear samples for assaying levels of various analytes in them such as lactoferrin, after the STT value is recorded at 5 min, leave the strip in place until wetting of at least $20 \mathrm{~mm}$ is obtained.

NOTE: If adequate wetting does not occur after DED has been induced, advance the strip deeper into the lower fornix to help reach this endpoint in reasonable time.

2. Cut the moistened strip and place immediately into $490 \mu \mathrm{L}$ of chilled Tear Collection Buffer ( $4 \% \mathrm{BSA}, 1 \mathrm{M} \mathrm{NaCl}, 0.1 \%$ Tween-20 in PBS with proteinase inhibitor cocktail).

3. Keep the samples on ice until they can be stored at $-80^{\circ} \mathrm{C}$, where they should remain until assayed.

6. Rose Bengal Staining (RBS)

1. Apply $50 \mu \mathrm{L}$ of $1 \%$ preservative-free lidocaine to the cornea using a micro-pipette.

2. After $30 \mathrm{~s}$, place $25 \mu \mathrm{L}$ of $1 \%$ rose bengal on the ocular surface and manually blink the eyelid to distribute it evenly.

3. Immediately start a timer.

4. At $3.5 \mathrm{~min}$, place a wire lid speculum between the lids.

5. At $4.0 \mathrm{~min}$, photograph the superior conjunctival and corneal surface (Figure 6).

NOTE: Adjust to the type of camera being used. Typical settings: digital single-lens reflex camera, aperture priority mode (aperture 13 or greater), ISO 6000, $100 \mathrm{~mm}$ macro lens attached with two $12.5 \mathrm{~mm}$ extension tubes, manual focus mode, lens at maximum 
magnification, and illumination from macro/ring flash set to automatic with through-the-lens mode. The ring flash's focus lamp is turned on to aid focus on the cornea.

6. Complete all photos for both eyes within $1 \mathrm{~min}$.

7. Score ocular surface staining using the NEI method ${ }^{23}$ modified as follows. Don not grade 6 separate conjunctival zones. Score the superior conjunctiva of each eye. This is the portion of the conjunctival surface easily photographed without manipulating the globe. Manipulation could artefactually change the staining of the ocular surface.

8. Tear lactoferrin levels are a surrogate measure of tear production from the lacrimal glands. Assay tear lactoferrin in tears collected as above by using an enzyme-linked immunosorbent assay ${ }^{24}$ kit following the instructions of the manufacturer.

\title{
5. Induction and treatment of dry eye
}

\author{
NOTE: Three portions of the orbital lacrimal gland system are injected.
}

1. Sedate the rabbits with acepromazine $0.2 \mathrm{mg} / \mathrm{kg}$ subcutaneously.

2. Shear off the fur in the periorbital and scalp area and completely remove any residual fur using Nair. Leave the skin entirely smooth for better visualization of the anatomical hallmarks and US-guided injection of concanavalin A (Figure 7).

3. Induce moderate sedation as described above.

4. Injection of the palpebral portion of the superior lacrimal gland (PSLG) NOTE: Perform injection of PSLG first.

1. Apply to the appropriate eye $25 \mu \mathrm{L}$ of preservative-free lidocaine $1 \%$ with a micropipette.

2. Evert the upper eyelid and apply gentle medial pressure to the posterior orbital rim until the protuberance marking the palpebral portion of the gland is seen. The PSLG appears as a small bulbous elevation in the posterior (temporal) portion of upper lid. NOTE: To view the gland tissue during the learning process, apply $5 \%$ fluorescein to the area (Figure $\mathbf{8 A}$ ). Tears can be seen streaming from the bulbous PSLG. Application of fluorescein is not needed for the administration of Con A; it is done only for illustration purposes to show the gland tissue.

3. Using fine-toothed forceps and a 27-gauge needle on a tuberculin syringe, directly penetrate the gland using a transconjunctival approach. Advance the needle $2 \mathrm{~mm}$ into the tissue and inject $500 \mu \mathrm{g}$ of Con A in a volume of $0.1 \mathrm{~mL}$ (Figure 8B). NOTE: This injection can sometimes be painful. If necessary, keep the animals under isoflurane until this injection is completed.

5. Injection of the orbital superior lacrimal gland (OSLG)

NOTE: OSLG follows in rapid succession.

1. Apply medial pressure to the globe causing the OSLG to protrude from the posterior incisure (see ref ${ }^{25}$ for anatomy, if needed). Apply medial pressure to the globe (Figure 9, red arrow) with the protuberance of the OSLG from the posterior incisure. The protuberance serves as gross localization to find the posterior incisure.

2. Use curved forceps with tips closed to indent the area until the bony opening in the skull is felt. This will be slit-like with an anterior/ posterior direction under the protuberance.

3. Apply modest pressure with forceps to leave an indentation in the skin, which will serve as the landmark for needle placement (Figure 10A).

4. Insert a needle (tuberculin syringe with a 27-gauge, 5/8-inch needle) perpendicular to the skin over the indentation mark (Figure 10B) $\sim 1 / 4$ inch into the incisure, then redirect the needle posteriorly and externally towards the lateral canthus aiming for the midpoint between the injection site and bony orbital rim.

NOTE: If the incisure is not precisely targeted with the needle, the skull blocks its advancement.

5. Once the hub of the needle is reached, slowly inject $1000 \mu \mathrm{g}$ of Con $\mathrm{A}$ in a volume of $0.2 \mathrm{~mL}$ (Figure 10C).

6. Complete the injection of both the PSLG and OSLG within 2-3 min.

7. Remove the animal from isoflurane sedation (if not yet done). Injection of the inferior lacrimal gland (ILG) can usually be completed without further sedation.

8. Injection of the inferior lacrimal gland

1. View the animal from the side. The prominence of the ILG can be seen along the lower anterior portion of the orbit (Figure 11A).

2. Draw a vertical line using a surgical marking pen or suitable permanent marker on the skin where the superficial part of the ILG gland transitions from its superficial (more external) resting place on the zygomatic bone to its more medial location in the orbit. This is typically inferior to the anterior limbus (Figure 11A).

3. Identify the end of the zygomatic bone by sweeping the vertically-held US probe across this line on the skin. The ILG transition occurs where the image of the gland changes from clearly circumscribed (hyperechoic line of the zygomatic bone is seen along the lower edge of the gland in the image) to one without a recognizable medial border (the zygomatic bone echo is no longer present, Figure 1).

4. Observe the relative position of the hand-piece to the line drawn on the skin when the US screen shows this change. This is the "injection site" where Con A should be given.

5. Control the depth of injection so as to place Con A into the gland at a point just medial to the zygomatic arch bone.

6. Determine the depth of injection as follows: Set the desired depth of injection as the depth of the zygomatic bone (hyperechoic signal) plus $1 \mathrm{~mm}$. Subtract this value from the known length of the needle (15 $\mathrm{mm}$ in this example).

7. Insert the needle into the gland at the "injection site" $\sim 12 \mathrm{~mm}$, then slowly withdraw it until the length of the exposed needle outside the body (measured with surgical calipers) is equal to the difference calculated in 5.8.6 (Figure 12). Inject $1000 \mu \mathrm{g}$ of Con $\mathrm{A}$ in $0.2 \mathrm{~mL}$. NOTE: To ensure that the capsule of the gland is pierced and not simply pushed by the needle, the needle should be inserted $\sim 12 \mathrm{~mm}$ or almost to the hub before its withdrawal begins.

8. Repeat the US to confirm the success of the injection. The ILG should show a characteristic hypoechoic space (Figure 1). NOTE: The ILG injection is the one best tolerated by the animals ${ }^{26}$ and is, therefore, done last.

9. Complete the entire procedure to inject all glands of both eyes within $10 \mathrm{~min}$. This will require achievement of competency in the procedure. NOTE: A single set of injections into the 2 orbital lacrimal glands will induce acute DED lasting 1-2 weeks. 
10. For DED of longer duration, inject Con A exactly as above every 7 days. Up to 6 such injections have been successfully performed.

\section{Post-procedure care}

1. Following injection of Con A, monitor the animals in their restraining bags for at least 10-20 min, or until the anesthetic effect has worn off.

2. Do not leave the animals unattended until they have regained sufficient consciousness to maintain sternal recumbency. Do not return them to their individual cages until they are fully recovered.

3. Post-procedure pain is usually mild and lasts less than $48 \mathrm{~h}$. Assess pain with the rabbit grimace scale. If needed, give a single dose of subcutaneous ketorolac ( $5 \mathrm{mg} / \mathrm{kg}$ ). For more severe pain, give subcutaneous buprenorphine $0.1 \mathrm{mg} / \mathrm{kg}$ every $8 \mathrm{~h}$.

\section{Representative Results}

Con A injections induced a strong inflammatory response in the lacrimal glands characterized by a dense lymphocytic infiltrate (Figure 13), accompanied by decreased tear production. All tear parameters were markedly altered (Table $\mathbf{1}$ and Table 2). In addition, tear lactoferrin levels were suppressed (control $=3.1 \pm 0.45 \mathrm{vs}$. Con $\mathrm{A}$ injected $=2.7 \pm 0.02 \mathrm{ng} / \mathrm{mg}$ protein $($ mean $\pm \mathrm{SEM}) ; \mathrm{p}<0.03)$. The end result was a compromised corneal and conjunctival epithelium, evidenced by increased rose bengal staining (Figure 6).

Injection of the three orbital LG tissues produced a consistent and uniform DED state unlike the states achieved by previous methods ${ }^{18,27}$. Key contributors to this result were the US-guided injection of the ILG and the injection of the OSLG. Table 1 summarizes the salient results of this method. All changes are consistent with severe DED.

A single set of Con A injections produces DED lasting about 1 week; all clinical parameters normalize by day 10 (Table 2). Sequential Con A injections about 1 week apart extend the duration of DED accordingly. For example, the second set of Con A injections on day 7 maintains DED for 2 weeks and so on. After approximately 5 sets of injections, the DED state often becomes permanent without the need for further injections.

When the rabbits with Con A-induced DED were treated with the novel agent phosphosulindac, it markedly suppressed the disease. For example, following one week's treatment with this agent TBUT increased markedly compared to vehicle-treated animals $(43.6 \pm 4.0$ vs. $12.2 \pm 2.8$ $\mathrm{s} ; \mathrm{p}<0.001$; mean \pm SEM respectively, for these and following values) while tear osmolarity was normalized $(294 \pm 4.6 \mathrm{vs}$. $311 \pm 2.0 \mathrm{mOsm} / \mathrm{L}$ $\mathrm{p}<0.002)$. Mechanistically, phosphosulindac decreased the levels of two crucial interleukins, IL-1 $\beta(8.4 \pm 1.2 \mathrm{vs} 21.2 \pm 6.6 \mathrm{pg} / \mathrm{mg} \mathrm{protein} ; \mathrm{p}<0.03)$ and IL-8 $(4.9 \pm 1.7 \text { vs. } 13.5 \pm 5.0 \mathrm{pg} / \mathrm{mg} \text { protein; } p<0.05)^{19}$. 


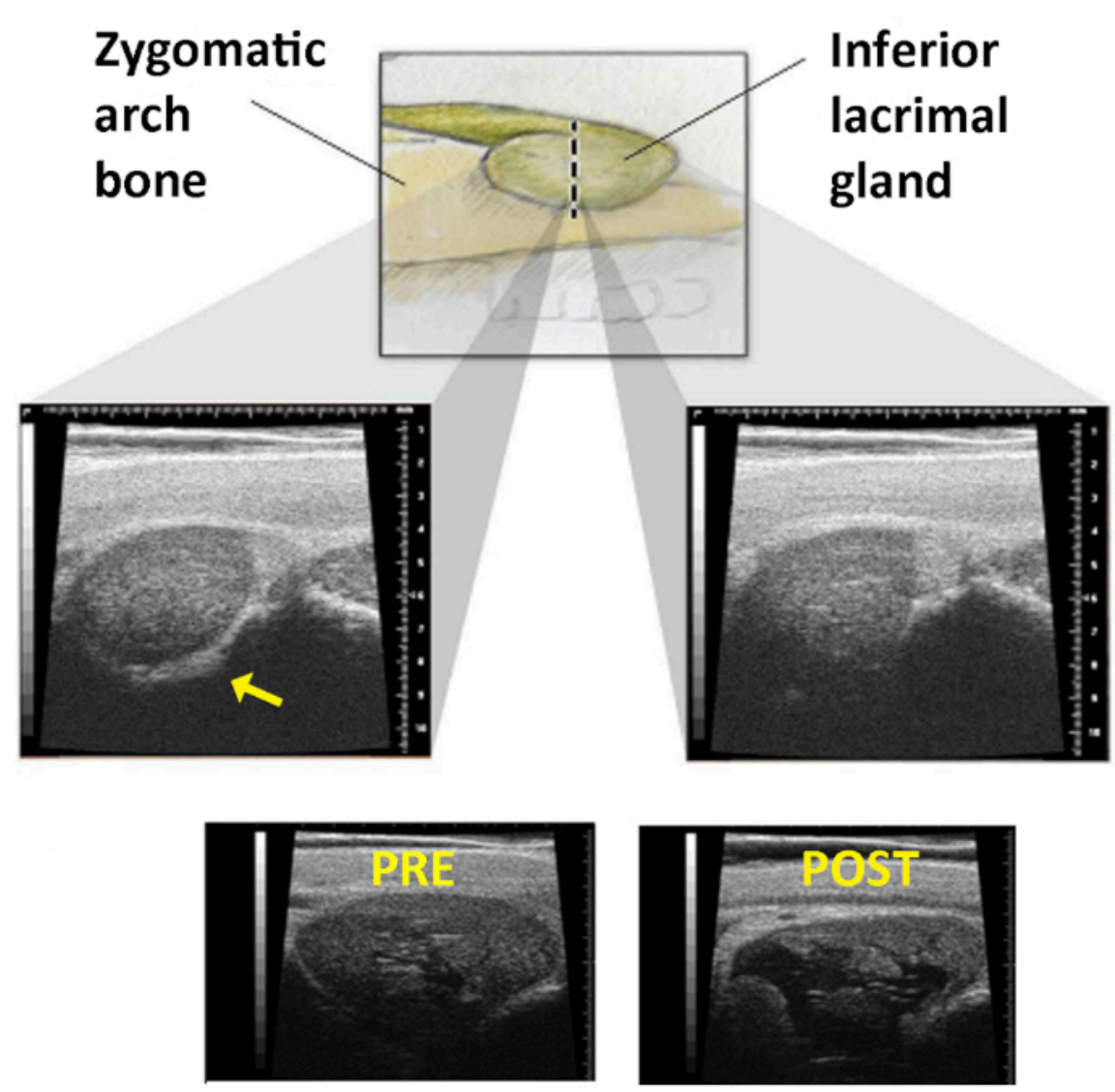

Figure 1: Ultrasound image of the inferior lacrimal gland. Upper Panel: The ILG as it moves deeper in orbit to lie beneath the zygomatic arch. The dashed line represents the line on skin across which the US probe is swept. Middle Panels: As the hand-piece is swept across this line, the examiner looks for loss of the zygomatic bone echo that is present in the left image (arrow) and disappears in the right. Lower Panels: Images of the ILG taken before (left) and after (right) injection of Con A. Development of a large cystic space within the gland confirms proper delivery. Reprinted with permission ${ }^{19}$. Please click here to view a larger version of this figure. 


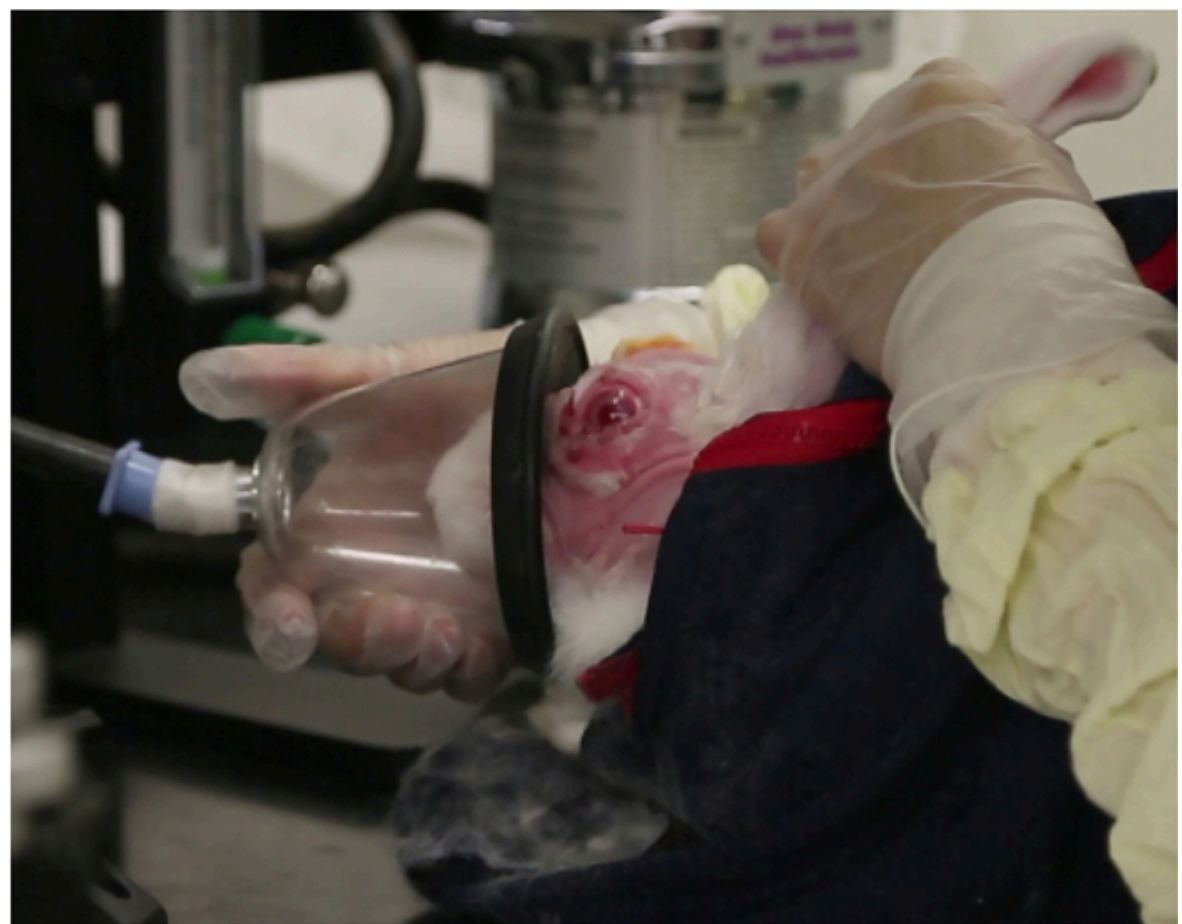

Figure 2: Gas mask sedation. This photograph shows the gas mask providing brief moderate sedation with isoflurane. Please click here to view a larger version of this figure. 

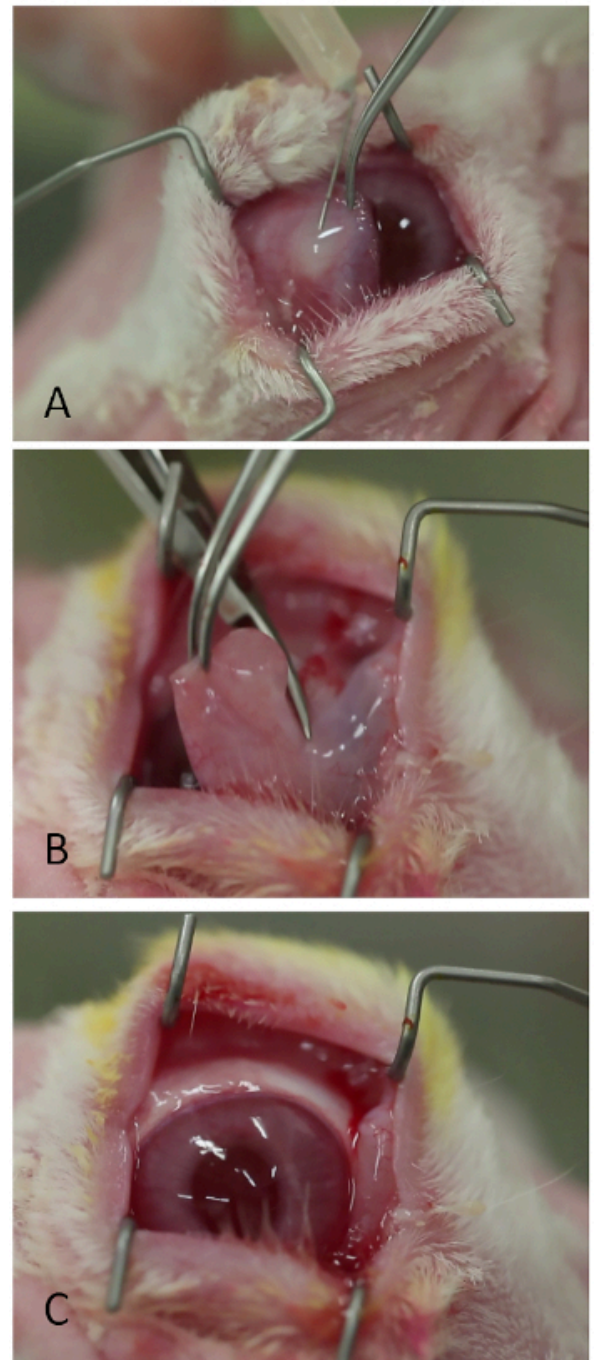

Figure 3: Removal of the nictitating membrane. (A) Injection of lidocaine/epinephrine. (B) Truncation of the nictitating membrane at its base with Westcott scissors. (C) The ocular surface visualized more readily after removal of the nictitating membrane. Please click here to view a larger version of this figure. 

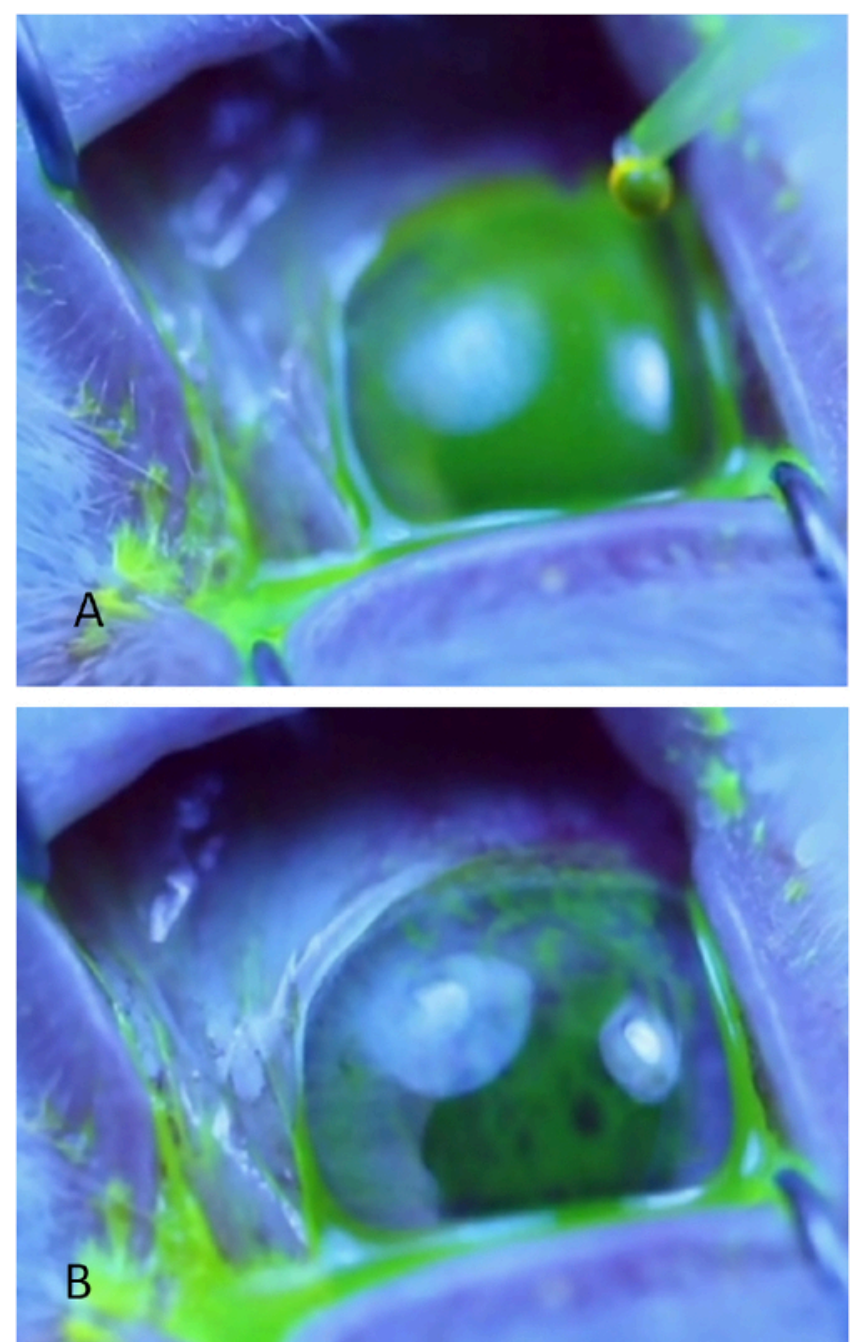

Figure 4: Tear break-up time measurement. (A) Uniform green tear film appearance of the corneal surface under blue light immediately following application of fluorescein drops. (B) Corneal surface that has already undergone marked break-up evidenced by multiple dark circles and linear streaks in the fluorescein. Break-up time is recorded as soon as the first dark spot or line develops. The two light blue circles are reflections of the light source off of the cornea. Please click here to view a larger version of this figure. 

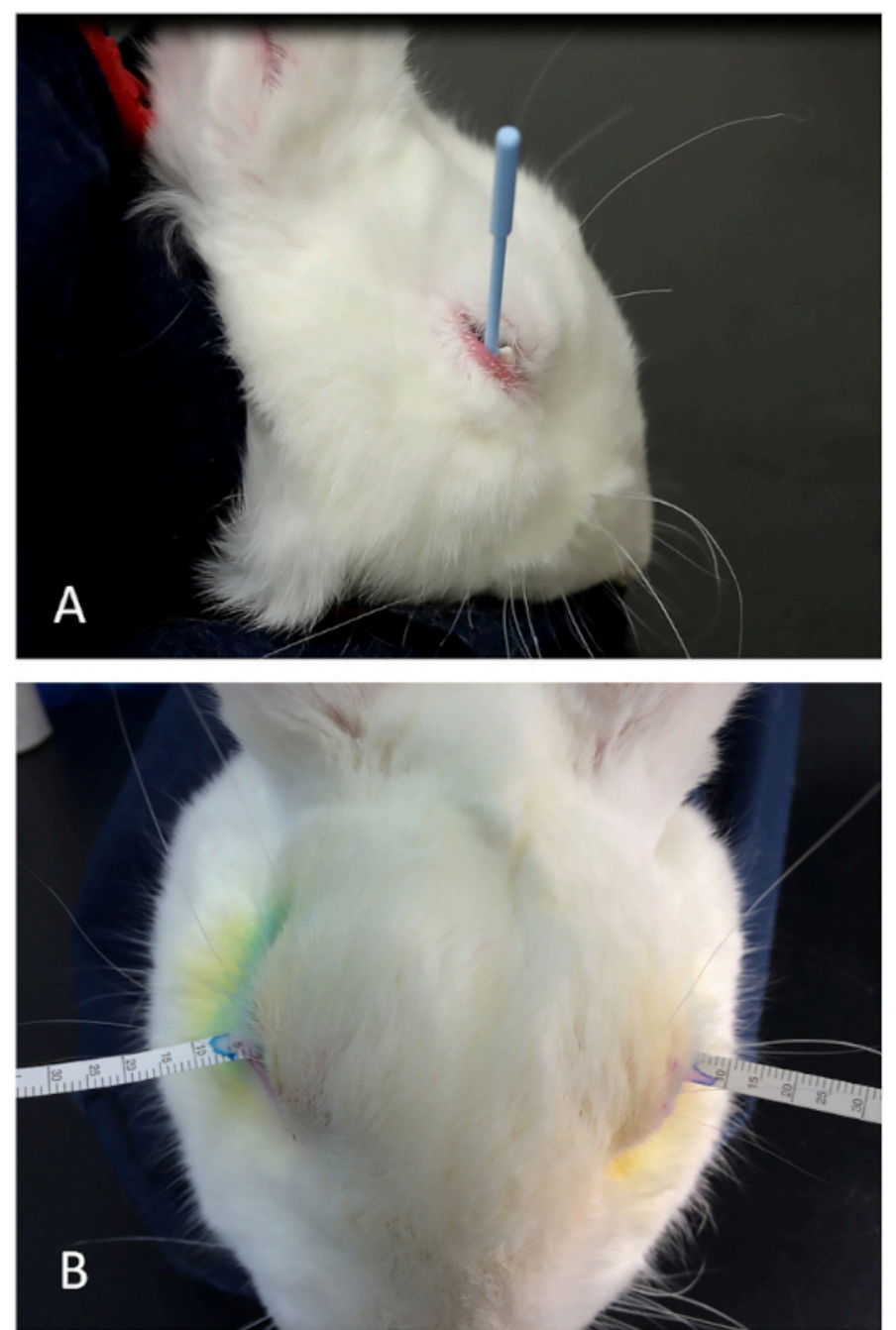

Figure 5: Schirmer's tear test. (A) The proper placement of the Weck-Cel sponge in the lower fornix to remove any residual topical lidocaine solution and baseline tears. By placing the posterior edge of the triangular sponge under the lower lid margin, one can maintain a very uniform technique to dry the ocular surface prior to placement of the tear strips. (B) A tear strip appropriately placed at the mid-position of the lower lid between the globe and lower lid (palpebral conjunctiva). Please click here to view a larger version of this figure. 

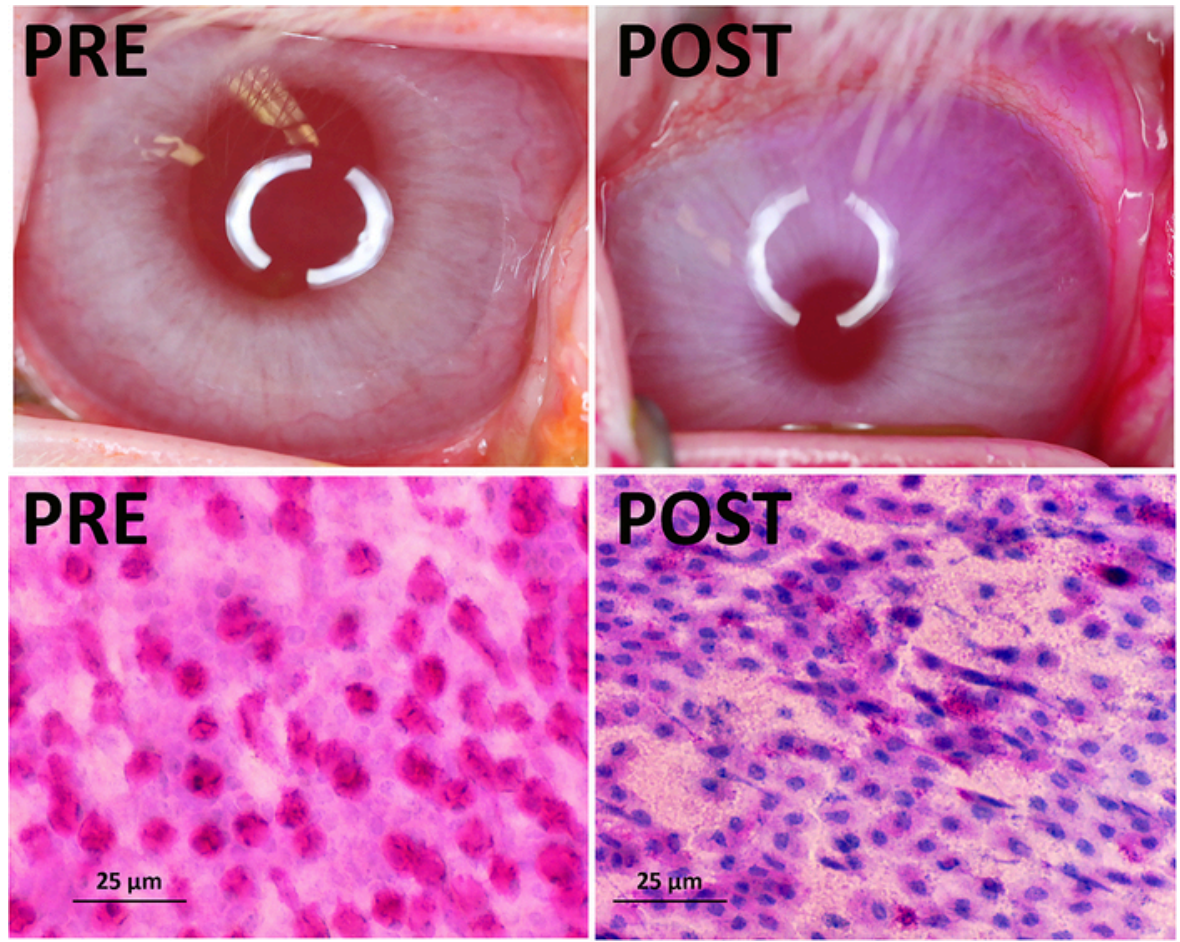

Figure 6: Rose bengal staining. Upper: Photographs of the corneal surface. Left: No rose bengal staining is present before treatment with Con A. Right: Diffuse corneal and conjunctival staining is seen in the upper nasal quadrant post-injection (upper right). Lower: Conjunctival impression cytology from the superior bulbar conjunctiva. Left: Numerous goblet cells are present before treatment. Right: Epithelial cells are present but goblet cells are absent post-treatment. Please click here to view a larger version of this figure. 

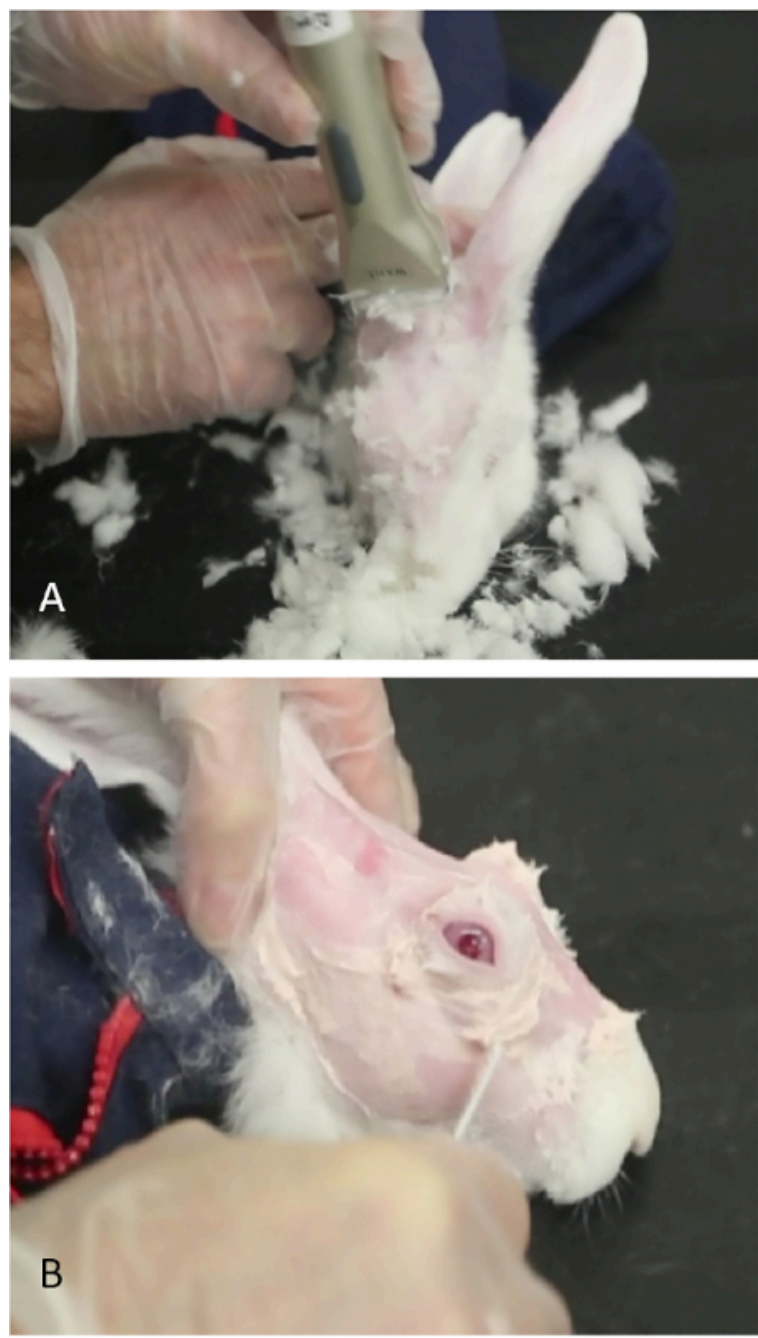

Figure 7: Preparation of rabbit for concanavalin A injections. (A) Small shears are used to remove fur, allowing easier visualization of landmarks to identify the orbital superior lacrimal gland. (B) Nair is used to remove hair that remains after shearing. Please click here to view a larger version of this figure. 


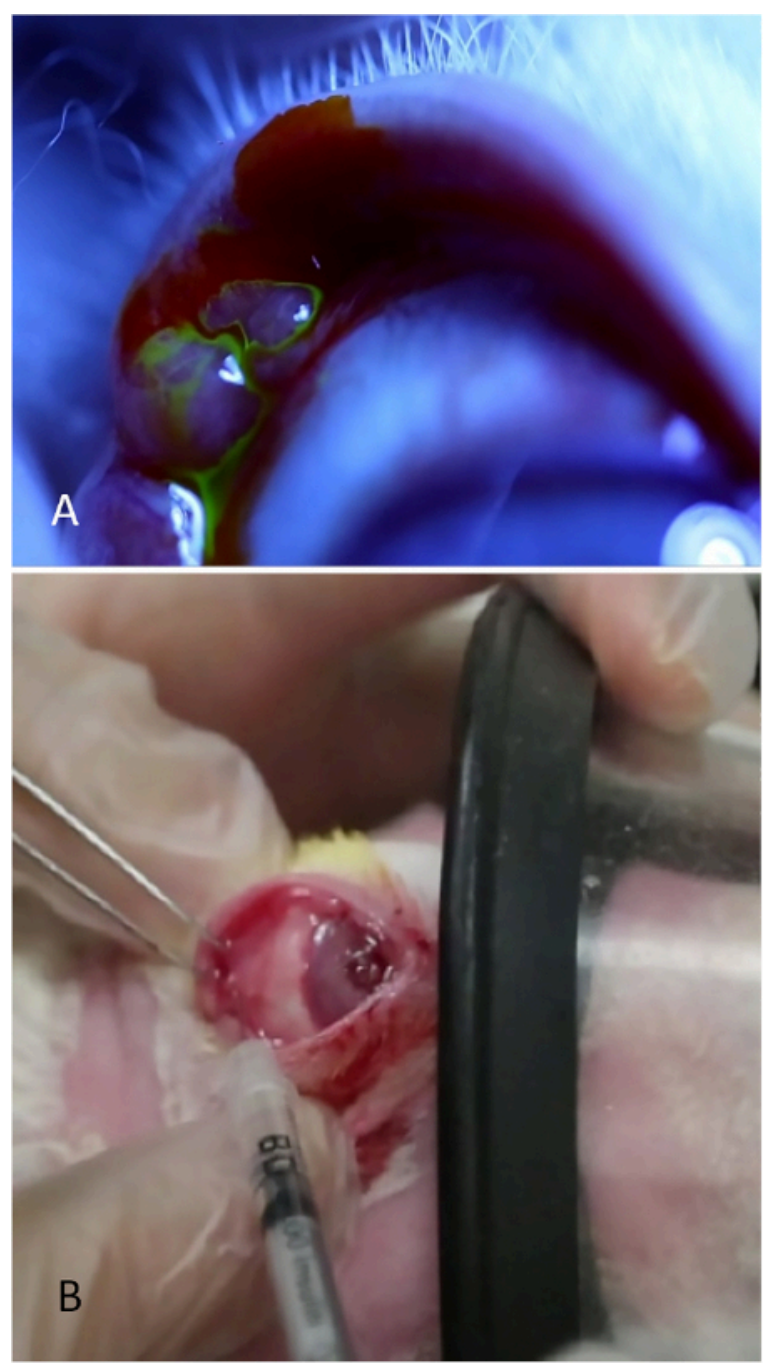

Figure 8: Injection of the palpebral lacrimal gland. (A) The palpebral lacrimal gland, appearing as a bulbous elevation in the posterior temporal portion of the upper lid. Tears are seen streaming from the surface of this gland after applying a drop of $2 \%$ fluorescein. (B) The palpebral lacrimal gland is being injected while the rabbit is receiving moderate sedation. One investigator retracts the eyelid, optimizes exposure of the gland, and secures the mask while the second investigator injects the gland. Please click here to view a larger version of this figure. 


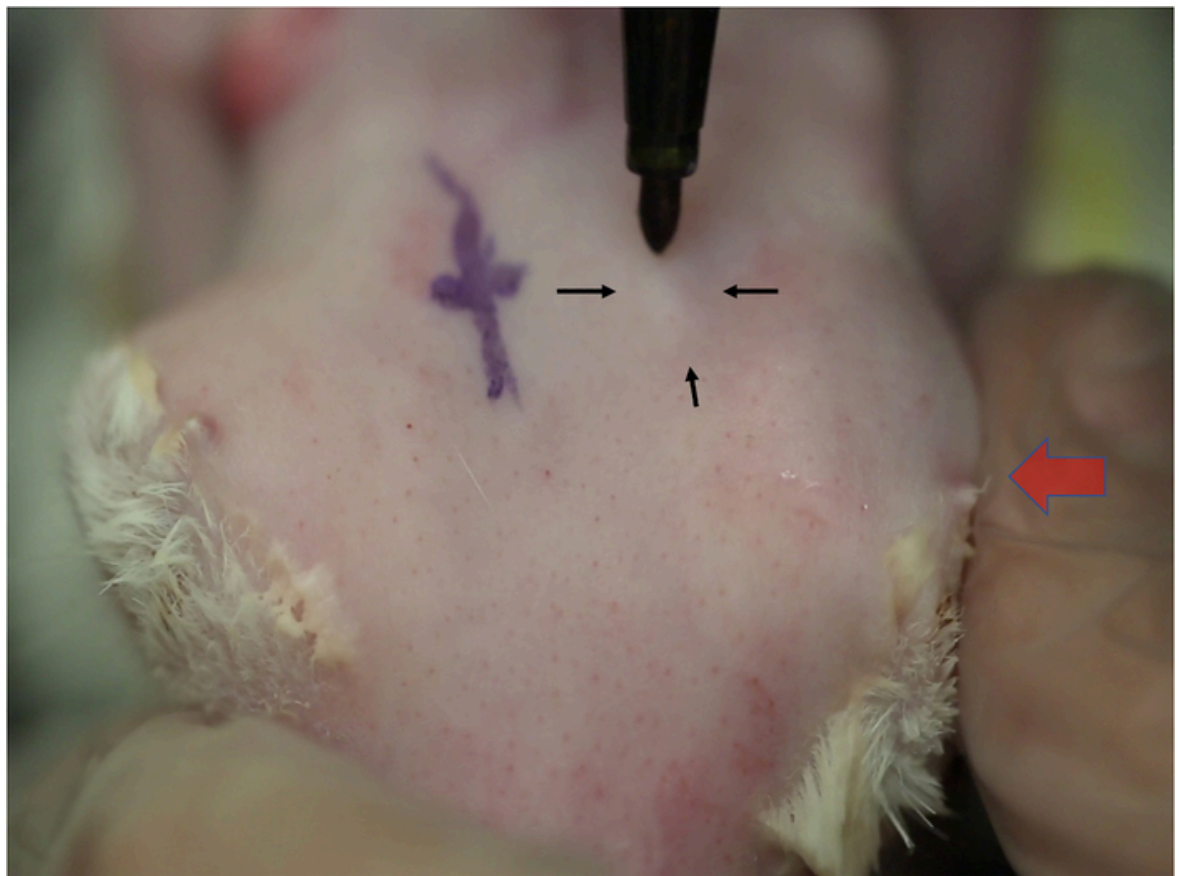

Figure 9: Localization of the orbital superior lacrimal gland. Changes in skin contours indicate the location of the OSLG as it protrudes through the posterior incisure. Alternating medial pressure on the globe (large arrow) causes the superior orbital gland to prolapse, which is seen as a small elevation in the skin. This elevation will increase in size each time the pressure is applied (small arrows). The location of this gland is usually in line with the posterior orbital rim. Please click here to view a larger version of this figure. 

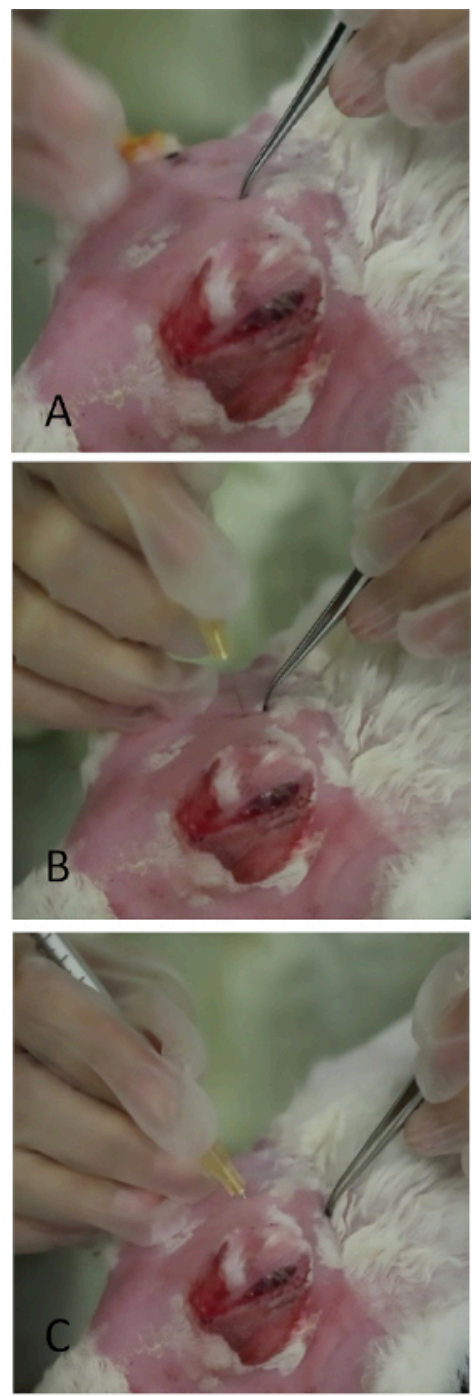

Figure 10: Injection of the orbital superior lacrimal gland. (A) Application of gentle pressure to the skull with fine-toothed forceps in the area which prolapsed as in Figure 9. A thin slit-like opening in the skull can be palpated. Leaving a small indentation mark with the forceps greatly aids placement of the needle during injection. (B) The needle is being inserted perpendicularly through the incisure. If placed incorrectly, its passage is stopped by the bony skull. (C) The needle is in final position angled towards the lateral canthus. Please click here to view a larger version of this figure. 

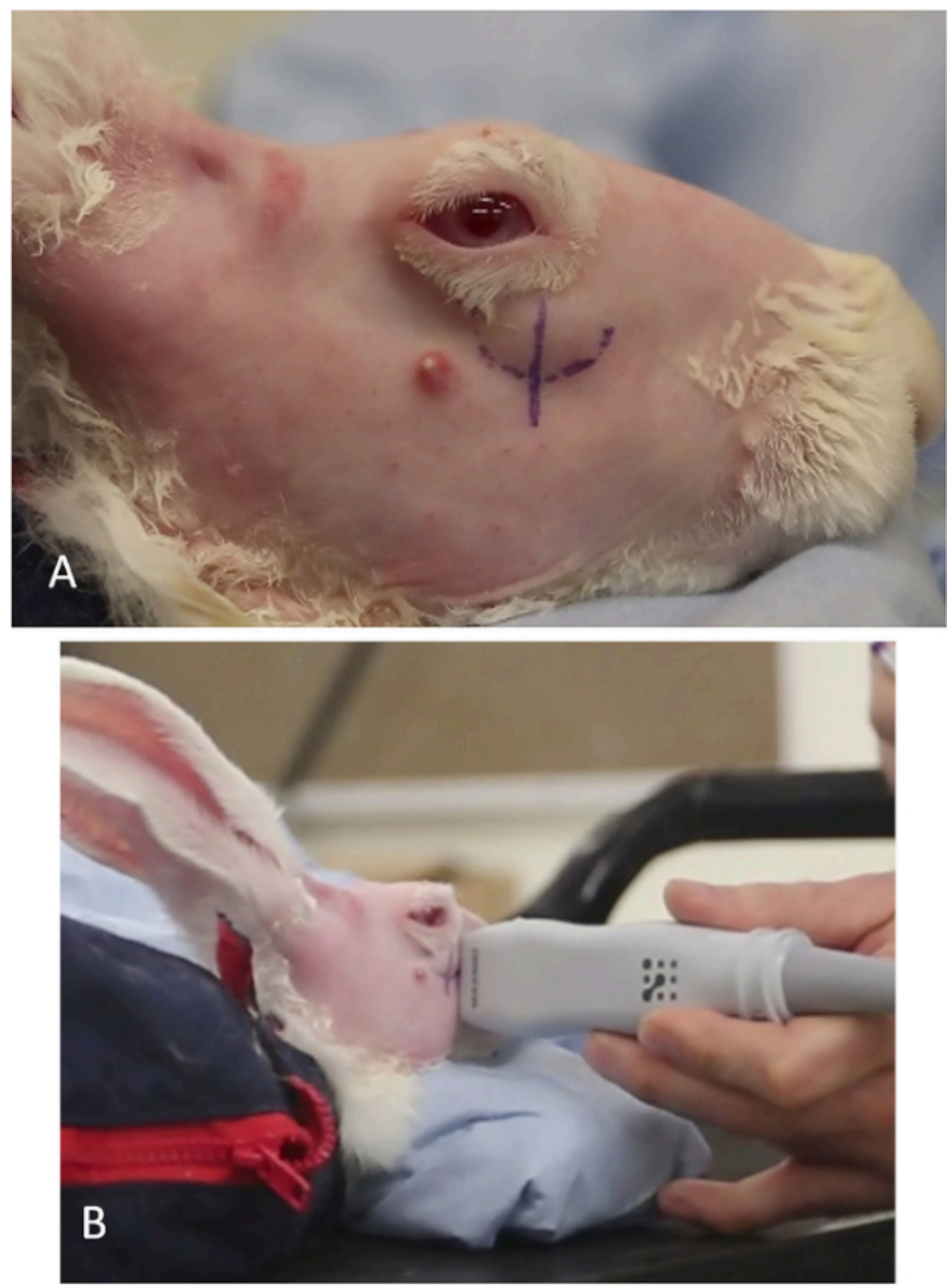

Figure 11: Localization of the inferior lacrimal gland. (A) The prominence of the superficial portion of the ILG seen through the lower lid. The curvilinear pen mark denotes the lower position of the gland. The vertical line, under the nasal limbus, denotes the approximate position where the ILG transitions to a deeper position within the orbit and serves as a visual reference for the US. (B) US hand-piece sweeping across the area of the vertical line; the US monitor will show where the zygomatic bone ends, where the ILG transitions and where the Con A injection should be given ("injection site"). Please click here to view a larger version of this figure. 


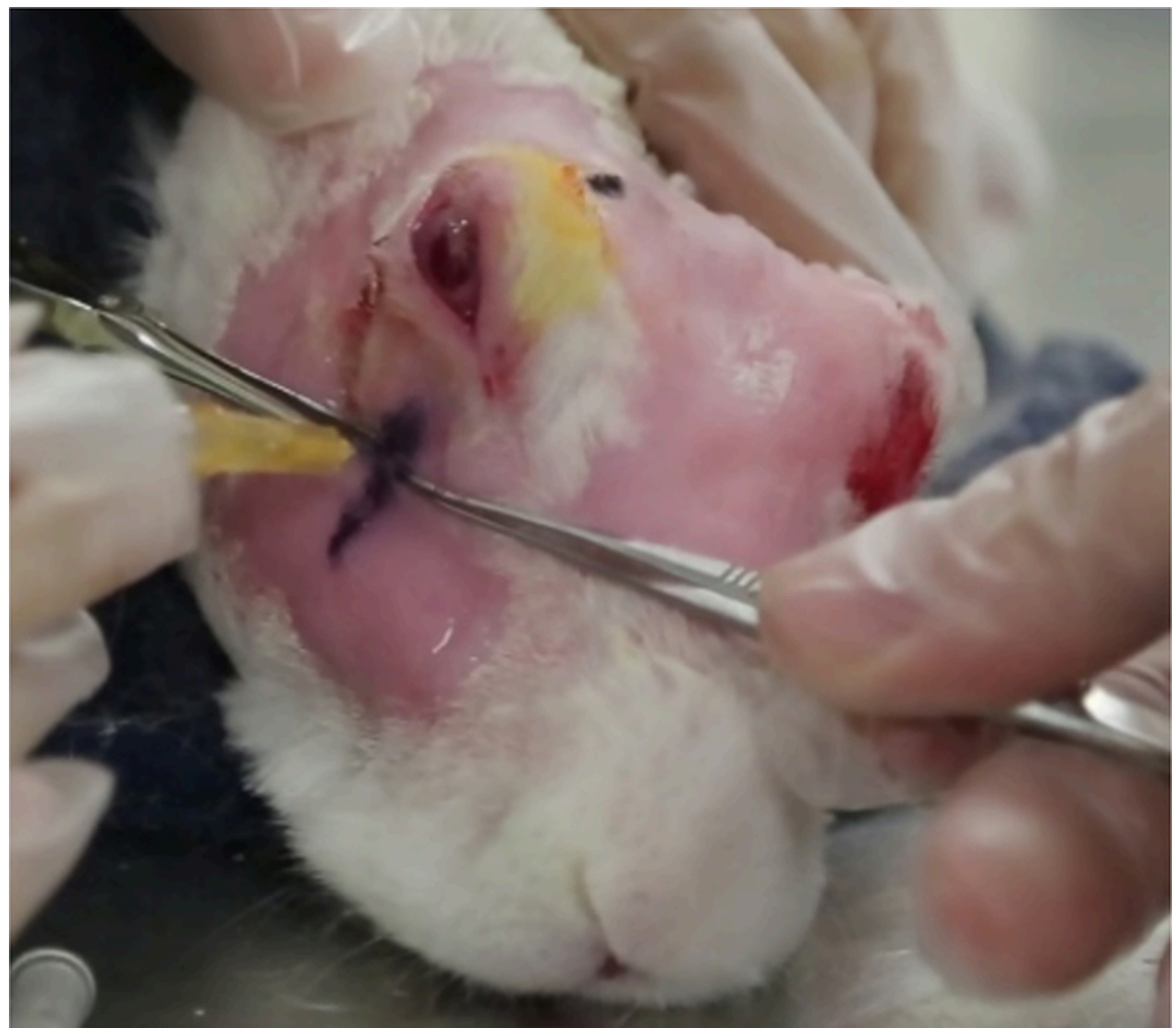

Figure 12: Injection of the inferior lacrimal gland. Injection of the ILG is done at the location identified by US. The depth of injection is calculated as described in the text (step 5.8.6). Calipers (seen behind the needle) ensure that the needle is placed at the proper depth before injection. Please click here to view a larger version of this figure.
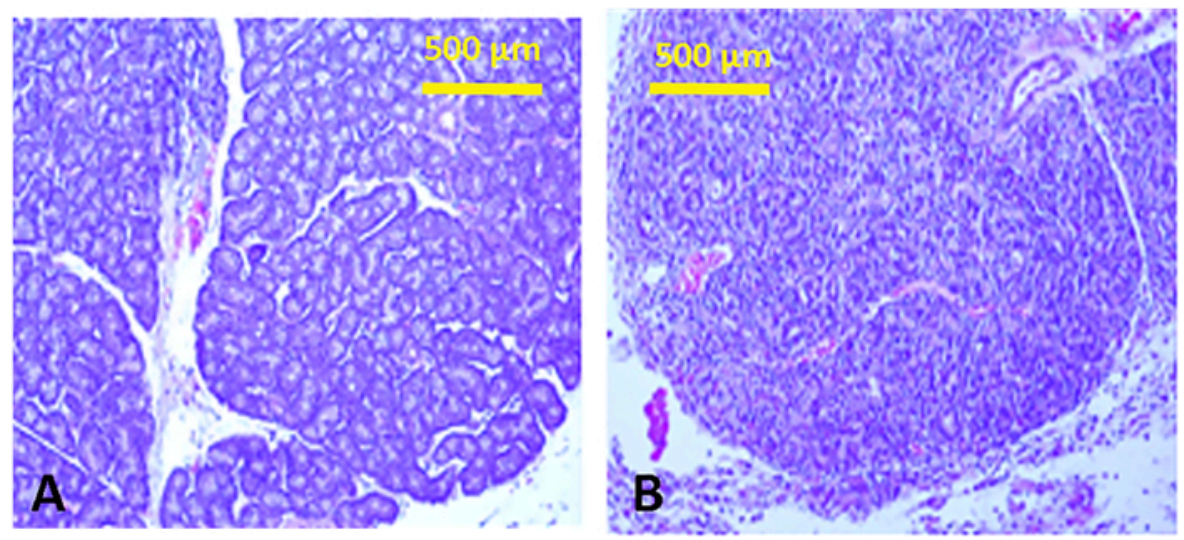

Figure 13: Histology of the lacrimal glands. Tissue sections of a normal inferior lacrimal gland with typical tubulo-alveolar structure (A) and following injection of Con A (B), showing marked lymphocytic infiltrate with effacement of structure. Similar inflammatory infiltrates are seen in the superior lacrimal glands. Please click here to view a larger version of this figure. 


\begin{tabular}{|c|c|c|c|c|c|c|}
\hline \multirow[t]{3}{*}{ Injection Method } & \multicolumn{2}{|l|}{ TBUT, sec } & \multicolumn{2}{|c|}{ Tear Osmolality, Osm/L } & \multicolumn{2}{|l|}{ STT, mm } \\
\hline & Baseline & Post-injection & Baseline & Post-injection & Baseline & Post-injection \\
\hline & \multicolumn{6}{|c|}{ mean \pm SEM, $\%$ change } \\
\hline $\begin{array}{l}\text { ILG without US } \\
\text { guidance }\end{array}$ & \multirow[t]{3}{*}{$58.5 \pm 1.5$} & $44.5 \pm 7.7$ & \multirow[t]{3}{*}{$297 \pm 4.9$} & $300 \pm 3.8$ & \multirow[t]{3}{*}{$15.2 \pm 0.9$} & $12.9 \pm 2.2$ \\
\hline \multirow[t]{2}{*}{ \%change } & & $-23 \%$ & & $1 \%$ & & $-15 \%$ \\
\hline & & $p=0.05$ & & $p=0.36$ & & $p=0.21$ \\
\hline $\begin{array}{l}\text { US-guided ILG + } \\
\text { PSLG }\end{array}$ & \multirow[t]{3}{*}{$59.4 \pm 0.6$} & $11.4 \pm 4.2$ & \multirow[t]{3}{*}{$296 \pm 4.7$} & $326 \pm 3.7$ & \multirow[t]{3}{*}{$15.1 \pm 1.3$} & $10.7 \pm 1.8$ \\
\hline \multirow[t]{2}{*}{$\%$ change } & & $-81 \%$ & & $10 \%$ & & $-29 \%$ \\
\hline & & $p<0.0001$ & & $p<0.0001$ & & $p<0.03$ \\
\hline $\begin{array}{l}\text { US-guided ILG + } \\
\text { PSLG + OSLG }\end{array}$ & \multirow[t]{3}{*}{$60 \pm 0.0$} & $6.2 \pm 1.3$ & \multirow[t]{3}{*}{$299 \pm 2.9$} & $309 \pm 2.8$ & \multirow[t]{3}{*}{$14.6 \pm 0.9$} & $9.9 \pm 1.3$ \\
\hline \multirow[t]{2}{*}{$\%$ change } & & $-90 \%$ & & $3 \%$ & & $-32 \%$ \\
\hline & & $p<0.0001$ & & $p<0.008$ & & $p<0.002$ \\
\hline
\end{tabular}

All values were measured on day 6 following a single injection of Con A into the glands listed. All comparisons were made to baseline. *ILG, inferior lacrimal gland; PSLG, palpebral portion of superior lacrimal gland; OSLG, orbital portion of superior lacrimal gland; US, ultrasound.

Table 1: Effect of injection technique and number of injection sites on dry eye severity.

\begin{tabular}{|c|c|c|c|c|}
\hline & \multirow[t]{2}{*}{ Baseline } & Day 6 & Day 13 & Day 21 \\
\hline & & 1 injection & 2 injections & 3 injections \\
\hline TBUT, sec & \multirow[t]{3}{*}{$58.5 \pm 1.5$} & $44.5 \pm 7.7$ & $29.2 \pm 7.8$ & $12.8 \pm 3.9$ \\
\hline \multirow[t]{2}{*}{$\%$ change } & & $-24 \%$ & $-50 \%$ & $-78 \%$ \\
\hline & & $p=0.17$ & $p=0.001$ & $p<0.0001$ \\
\hline TOsm, Osm/L & \multirow[t]{3}{*}{$297 \pm 4.9$} & $300 \pm 3.9$ & $308 \pm 4.9$ & $313 \pm 2.7$ \\
\hline \multirow[t]{2}{*}{$\%$ change } & & $1 \%$ & $4 \%$ & $5 \%$ \\
\hline & & $p=0.36$ & $p=0.04$ & $p=0.003$ \\
\hline $\mathrm{STT}, \mathrm{mm}$ & \multirow[t]{3}{*}{$15.2 \pm 0.9$} & $9.3 \pm 1.6$ & $12.9 \pm 1.6$ & $7.4 \pm 1.1$ \\
\hline \multirow[t]{2}{*}{$\%$ change } & & $-39 \%$ & $-15 \%$ & $-51 \%$ \\
\hline & & $p=0.17$ & $p=0.13$ & $p=0.008$ \\
\hline
\end{tabular}

Table 2: Effect of repeated Con A injections into ILG on duration of DED.

\section{Discussion}

Rabbits are highly attractive for the study of DED. Their cornea and conjunctiva have a surface area closer to that of humans compared to mice and rats; their complement of drug metabolizing enzymes such as esterases, and histology of their lacrimal glands are similar to those of humans, and their eyes are large enough for informative pharmacokinetic studies. Compared to pigs and monkeys, with which they share similar features, they cost less and their experimental manipulation is easier. If mechanistic studies are contemplated, a relative drawback of the rabbit, compared to mice, is that fewer reagents (e.g., monoclonal antibodies) are available. On the other hand, the rabbit is far superior to mice for pharmacokinetic and biodistribution studies because individual tissues are easily dissected and of sufficient size for analytical work, avoiding "sample pooling."

A critical general parameter is the acclimation period of the rabbits. The animals are shipped from the vendor under conditions that often do not ensure a transportation environment of the appropriate temperature or humidity. Some animals may have already developed dry eye upon arrival. A two-week period of acclimation is recommended. Equally important is scrupulous attention to the humidity and temperature of the space where the study rabbits are housed in the vivarium. Deviations in either condition can induce huge variations in their eye status. Have back-up humidifiers and dehumidifiers on hand. If the central system fails, act quickly to restore ambient humidity using the back-up equipment. Bear in mind that such unfortunate developments are more common in the summer months. The three most critical steps, however, for successfully inducing DED in rabbits are: 1) the skillful use of US imaging to identify the ILG and to direct and confirm injection of Con A; 2) ensuring injection of both the ILG and the two parts of the SLG; and 3) reliably and reproducibly assaying the parameters of DED.

Developing the required experimental skill is not trivial but should not deter any serious investigator. Expect the learning curve to be completed within five iterations. An US imaging system of reasonable quality is essential. Recognition of anatomical hallmarks by US is important, therefore, the investigator should review the rabbit anatomy. The excellent description of rabbit anatomy by Davis ${ }^{25}$, a classic, can be immensely helpful. 
Also keep in mind the variation in the size of the ILG. The corollary to this is that the success of Con A must always be confirmed with follow up imaging. Variations in the response to Con A in a group of rabbits is most often due to the injection technique (unsuccessful or partially successful injection) or to ignoring the capacity of residual lacrimal gland tissues to compensate with overproduction of tears. For those who wish to master the injection technique, injecting methylene blue followed by prompt anatomic dissection can be helpful; visualization is achieved if it reaches the lacrimal gland or spills onto neighboring tissues. To date, this injection method has been performed over 270 times by the authors without a single complication.

Assaying the five parameters of DED presented above can be as tricky as is their determination in clinical practice. Although circadian variations have not yet been formally documented in any of them, there is enough background evidence of such phenomena in the eye ${ }^{28}$ that they should be assayed at the same time of day $( \pm 1 \mathrm{~h})$, especially when repeat assays are to be performed and compared to each other. Consistency in performing these assays is essential. A team of two is required. Four or more investigators in the same room participating in the assays can be disruptive, given that some steps require strict timing. Appropriate and high-quality photographic documentation, where indicated, is important.

This model is ideally suited for drug development studies. Mastery of the animal model and assay techniques ensured excellent reproducibility ${ }^{19}$ of efficacy and safety studies.

This is a powerful experimental approach because it eliminates the confounding variability of prior models, has streamlined the animal model and essentially standardized assaying the five parameters of DED. The successful application of this model to the study of a candidate therapeutic agent has affirmed its practical utility as an informative animal model for a disease in desperate need of novel agents and of a deeper understanding of its pathogenesis.

\section{Disclosures}

The authors declare no competing interests except for BR who has an equity position in Medicon Pharmaceuticals, Inc. and Apis Therapeutics, LLC; and LH, an employee of Medicon Pharmaceuticals, Inc. with an equity position in Apis Therapeutics, LLC.

\section{Acknowledgments}

All animal studies were completed in accordance and compliance with all relevant regulatory and institutional guidelines. All studies were approved by the Institutional Review Board of Stony Brook University and performed in accordance with the ARVO Statement for the Use of Animals in Ophthalmic and Vision Research.

These studies were supported in part by a Targeted Research Opportunities grant from the Stony Brook University School of Medicine (Grant Number 1149271-1-82502) and a research grant from Medicon Pharmaceuticals, Inc., Setauket, NY. The authors thank Michele McTernan for editorial support.

\section{References}

1. Paulsen, A. J. et al. Dry eye in the Beaver Dam Offspring Study: prevalence, risk factors, and health-related quality of life. American Journal of Ophthalmology. 157 (4), 799-806 (2014).

2. Vehof, J., Kozareva, D., Hysi, P. G., \& Hammond, C. J. Prevalence and risk factors of dry eye disease in a British female cohort. British Journal of Ophthalmology. 98 (12), 1712-1717 (2014).

3. Tan, L. L., Morgan, P., Cai, Z. Q., \& Straughan, R. A. Prevalence of and risk factors for symptomatic dry eye disease in Singapore. Clinical and Experimental Optometry. 98 (1), 45-53 (2015).

4. Craig, J. P. et al. TFOS DEWS II Report Executive Summary. The Ocular Surface. 15 (4), 802-812 (2017).

5. Baudouin, C. et al. Clinical impact of inflammation in dry eye disease: proceedings of the ODISSEY group meeting. Acta Ophthalmologica (Copenhagen). 96 (2), 111-119 (2018).

6. Calonge, M. et al. Dry eye disease as an inflammatory disorder. Ocular Immunology and Inflammation. 18 (4), $244-253$ (2010).

7. Pflugfelder, S. C., \& de Paiva, C. S. The Pathophysiology of Dry Eye Disease: What We Know and Future Directions for Research. Ophthalmology. 124 (11S), S4-S13 (2017).

8. Buckley, R. J. Assessment and management of dry eye disease. Eye (London, England). 32 (2), $200-203$ (2018).

9. Clayton, J. A. Dry Eye. New England Journal of Medicine. 378 (23), 2212-2223 (2018).

10. Barabino, S. Animal models of dry eye. Archivos de la Sociedad Española de Oftalmología. 80 (12), 693-694; 695-696 (2005).

11. Stern, M. E., \& Pflugfelder, S. C. What We Have Learned From Animal Models of Dry Eye. International Ophthalmology Clinics. 57 (2), 109-118 (2017).

12. Chen, Z. Y., Liang, Q. F., \& Yu, G. Y. Establishment of a rabbit model for keratoconjunctivitis sicca. Cornea. 30 (9), $1024-1029$ (2011).

13. Gilbard, J. P., Rossi, S. R., \& Gray, K. L. A new rabbit model for keratoconjunctivitis sicca. Investigative Ophthalmology \& Visual Science. 28 (2), 225-228 (1987).

14. Guo, Z. et al. Autologous lacrimal-lymphoid mixed-cell reactions induce dacryoadenitis in rabbits. Experimenal Eye Research. 71 (1), $23-31$ (2000).

15. Burgalassi, S., Panichi, L., Chetoni, P., Saettone, M. F., \& Boldrini, E. Development of a simple dry eye model in the albino rabbit and evaluation of some tear substitutes. Ophthalmic Research. 31 (3), 229-235 (1999).

16. Xiong, C. et al. A rabbit dry eye model induced by topical medication of a preservative benzalkonium chloride. Investigative Ophthalmology \& Visual Science. 49 (5), 1850-1856 (2008).

17. Heymann, F., Hamesch, K., Weiskirchen, R., \& Tacke, F. The concanavalin A model of acute hepatitis in mice. Lab Animal. 49 (1 Suppl), $12-20$ (2015). 
18. Nagelhout, T. J., Gamache, D. A., Roberts, L., Brady, M. T., \& Yanni, J. M. Preservation of tear film integrity and inhibition of corneal injury by dexamethasone in a rabbit model of lacrimal gland inflammation-induced dry eye. Journal of Ocular Pharmacology and Therapeutics. 21 (2), 139-148 (2005).

19. Honkanen, R. A., Huang, L., Xie, G., \& Rigas, B. Phosphosulindac is efficacious in an improved concanavalin A-based rabbit model of chronic dry eye disease. Translational Research. 198 58-72 (2018).

20. Huang, L. et al. The novel phospho-non-steroidal anti-inflammatory drugs, OXT-328, MDC-22 and MDC-917, inhibit adjuvant-induced arthritis in rats. British Journal of Pharmacology. 162 (7), 1521-1533 (2011).

21. Mattheolabakis, G. et al. Topically applied phospho-sulindac hydrogel is efficacious and safe in the treatment of experimental arthritis in rats. Pharmaceutical Research. 30 (6), 1471-1482 (2013).

22. Osmalek, T., Froelich, A., \& Tasarek, S. Application of gellan gum in pharmacy and medicine. International Journal of Pharmaceutics. 466 (1-2), 328-340 (2014).

23. Lemp, M. A. Report of the National Eye Institute/Industry workshop on Clinical Trials in Dry Eyes. Contact Lens Association of Ophthalmologists Journal. 21 (4), 221-232 (1995).

24. Dal Piaz, F., Braca, A., Belisario, M. A., \& De Tommasi, N. Thioredoxin system modulation by plant and fungal secondary metabolites. Current Medicinal Chemistry. 17 (5), 479-494 (2010).

25. Davis, F. A. The Anatomy and Histology of the Eye and Orbit of the Rabbit. Transactions of the American Ophthalmological Society. 27400 402-441 (1929).

26. Lima, L., Lange, R. R., Turner-Giannico, A., \& Montiani-Ferreira, F. Evaluation of standardized endodontic paper point tear test in New Zealand white rabbits and comparison between corneal sensitivity followed tear tests. Veterinary Ophthalmology. 18 (Suppl 1), 119-124 (2015).

27. Zheng, W. et al. Therapeutic efficacy of fibroblast growth factor 10 in a rabbit model of dry eye. Mol Med Report. 12 (5), $7344-7350$ (2015).

28. Wiechmann, A. F., \& Summers, J. A. Circadian rhythms in the eye: the physiological significance of melatonin receptors in ocular tissues. Progress in Retinal and Eye Research. 27 (2), 137-160 (2008). 